\title{
Enantioselective Cu-Catalyzed Conjugate Addition of Alkylzinc Reagents to Acyclic Aliphatic Enones
}

\author{
Andrew P. Duncan and James L. Leighton* \\ Department of Chemistry, Columbia University, New York, New York, 10027 \\ Supporting Information
}

General Information. All reactions were carried out under an atmosphere of nitrogen in flame- or oven-dried glassware with magnetic stirring unless otherwise indicated. Air-sensitive compounds were manipulated using standard Schlenk and glovebox techniques. All reaction solvents were purified by degassing with argon and passage through an activated alumina column. Copper(II) trifluoromethanesulfonate, cyclohexylphosphine, (S)-tert-leucinol, trifluoromethanesulfonic anhydride, benzylidene acetone, and trans-3-nonen-2-one were obtained from Aldrich and used without further purification. Other enones were synthesizes according to known methods. Triethylamine was distilled from $\mathrm{CaH}_{2}$ prior to use. Trifluoroethanol was distilled from $\mathrm{CaSO}_{4}$ and freeze-pump-thaw degassed (1x) prior to use. Diethylzinc and dimethylzinc were obtained from Strem and used without further purification. ${ }^{1} \mathrm{H}$ NMR spectra were recorded on Bruker DPX-300 (300 MHz) or DPX-400 (400 MHz) spectrometers. ${ }^{1} \mathrm{H}$ NMR chemical shifts $(\square)$ are reported in parts per million (ppm) relative to residual protiated solvent $\left(\mathrm{CDCl}_{3}, 7.25 ; \mathrm{C}_{6} \mathrm{D}_{6}, 7.15\right)$. Data are reported as follows: $(\mathrm{s}=$ singlet, $\mathrm{d}=$ doublet, $\mathrm{t}=$ triplet, $\mathrm{q}=$ quartet, quint $=$ quintet, $\mathrm{m}=$ multiplet, $\mathrm{dt}=$ doublet of triplets, $\mathrm{dd}=$ doublet of doublets, $\mathrm{ddd}=$ doublet of doublet of doublets; coupling constant(s) in Hz; integration; assignment). Proton decoupled ${ }^{13} \mathrm{C}$ NMR spectra were recorded on a Bruker DPX-300 (75 MHz) and are reported in ppm from $\mathrm{CDCl}_{3}$ internal standard $(77.0 \mathrm{ppm}) .{ }^{13} \mathrm{C}$ resonances are singlets, unless otherwise specified. ${ }^{31} \mathrm{P}$ NMR spectra were recorded on a Bruker DPX-300 (121.6 MHz) and are reported in ppm from $\mathrm{H}_{3} \mathrm{PO}_{4}\left(85 \%\right.$ in $\left.\mathrm{H}_{2} \mathrm{O}\right)$ external standard (0.0 ppm). ${ }^{19} \mathrm{~F}$ NMR spectra were recorded on a Bruker DPX-300 (282 MHz) and are reported in ppm from internal $\square, \square, \square$-trifluorotoluene $(0.00 \mathrm{ppm})$. Infrared spectra were recorded on a Perkin Elmer Paragon 1000 FT-IR spectrometer. Gas chromatographic analyses were performed on a Hewlett-Packard 6890 Series Gas Chromatograph equipped with a capillary split-splitless inlet and flame 
ionization detector with electronic pneumatics control using either a Supelco B-Dex 120 (30 m x 0.25 $\mathrm{mm}$ ) capillary GLC column or an Astec Chiraldex G-TA (30 m x $0.25 \mathrm{~mm}$ x $0.125 \mu \mathrm{m}$ ) capillary GLC column with Astec Retention Gap Guard Column (5m x 0.25mm). Optical rotations were recorded on a JASCO DIP-1000 digital polarimeter; the concentration $c$ is reported in $\mathrm{g} / 100 \mathrm{~mL}$. Low resolution mass spectra were obtained on a JEOL HX110 mass spectrometer in the Columbia University Mass Spectrometry Laboratory.

Preparation of ligand 2d: To a cooled $\left(-78{ }^{\circ} \mathrm{C}\right)$ solution of cyclohexylphosphine $(200 \square \mathrm{L}, 1.51$ mmol, 1.0 equiv) in THF (10 mL) was added $n$-BuLi (2.5 M in hexanes, $0.60 \mathrm{~mL}, 1.51 \mathrm{mmol}, 1.0$ equiv). The resulting solution of lithium cyclohexylphosphide was allowed to warm to ambient temperature over 30 min, and was subsequently re-cooled to $-78{ }^{\circ} \mathrm{C}$. A solution of $(S)-N-[3,5-$ bis(trifluoromethyl)benzenesulfonyl]-2-tert-butylaziridine (0.350 g, $1.51 \mathrm{mmol}, 1.0 \mathrm{equiv})$ in THF was added via syringe. The reaction mixture was allowed to warm slowly to ambient temperature overnight $(10 \mathrm{~h})$. The resulting solution of lithiated phosphine sulfonamide $1 \mathbf{d}$ was then re-cooled $\left(-78^{\circ} \mathrm{C}\right)$ and $n$ BuLi (2.5 M in hexanes, $0.60 \mathrm{~mL}, 1.51 \mathrm{mmol}, 1.0$ equiv) was added. The resulting clear, pale yellow solution was maintained at $-78^{\circ} \mathrm{C}$ for $2 \mathrm{~h}$. Another portion of the aziridine $(0.350 \mathrm{~g}, 1.51 \mathrm{mmol}, 1.0$ equiv) was then added, and the reaction mixture was allowed to warm slowly to ambient temperature overnight (10 h). Acetic acid (175 $\square \mathrm{L}, 3.02 \mathrm{mmol}, 2.0$ equiv) was then added dropwise via syringe, causing the immediate formation of a precipitate. Volatile materials were removed in vacuo, affording unpurified $\mathbf{2 d}$ as a colorless, viscous oil. While maintaining a nitrogen atmosphere, the oil was extracted with $\mathrm{CH}_{2} \mathrm{Cl}_{2}(15 \mathrm{~mL})$. The resulting suspension was filtered through a short pad of dry silica gel in a medium porosity fritted funnel. The silica pad was washed with several additional portions of $\mathrm{CH}_{2} \mathrm{Cl}_{2}$ to ensure complete elution of the phosphine bis(sulfonamide). The combined $\mathrm{CH}_{2} \mathrm{Cl}_{2}$ portions were concentrated to afford pure phosphine bis(sulfonamide) $2 \mathbf{d}$ as a white powder (0.758 g, $1.30 \mathrm{mmol}, 86 \%)$. ${ }^{1} \mathrm{H}$ NMR $\left(\mathrm{C}_{6} \mathrm{D}_{6}, 300 \mathrm{MHz}\right), \square 6.52$ (br s, $\left.1 \mathrm{H}, \mathrm{NH}\right), 6.15$ (d, 1H, J = $\left.10 \mathrm{~Hz}, \mathrm{NH}\right), 3.58$ (m, 1H, NCH), 3.42 $(\mathrm{m}, 1 \mathrm{H}, \mathrm{NCH}), 1.87-1.95,1.02-1.69\left(\mathrm{~m}, 15 \mathrm{H}\right.$, both of $\mathrm{PCH}_{2}$ and $\left.\mathrm{C}_{6} \mathbf{H}_{11}\right), 0.86\left(\mathrm{~s}, 9 \mathrm{H},\left(\mathrm{CH}_{3}\right)_{3} \mathrm{C}\right), 0.82(\mathrm{~s}$, 9H, $\left.\left(\mathrm{CH}_{3}\right)_{3} \mathrm{C}\right) \mathrm{ppm} .{ }^{13} \mathrm{C}\left\{{ }^{1} \mathrm{H}\right\} \mathrm{NMR}\left(\mathrm{C}_{6} \mathrm{D}_{6}, 75 \mathrm{MHz}\right) \square 120.2(\mathrm{q}, J=319 \mathrm{~Hz}),, 119.8(\mathrm{q}, J=319 \mathrm{~Hz}), 66.2$ $(\mathrm{d}, J=23 \mathrm{~Hz}), 63.9(\mathrm{~d}, J=12 \mathrm{~Hz}), 37.4(\mathrm{~d}, J=11 \mathrm{~Hz}), 35.7,35.5(\mathrm{~d}, J=7 \mathrm{~Hz}), 31.9(\mathrm{~d}, J=18 \mathrm{~Hz}), 30.3$ 
$(\mathrm{d}, J=13 \mathrm{~Hz}), 29.3(\mathrm{~d}, J=12 \mathrm{~Hz}), 28.1(\mathrm{~d}, J=19 \mathrm{~Hz}), 27.6(\mathrm{~d}, J=6 \mathrm{~Hz}), 27.1(\mathrm{~d}, J=7 \mathrm{~Hz}), 27.0(\mathrm{~d}, J=$ $6 \mathrm{~Hz}), 26.5,26.4$ ppm. ${ }^{31} \mathrm{P}\left\{{ }^{1} \mathrm{H}\right\}$ NMR $\left(\mathrm{C}_{6} \mathrm{D}_{6}, 121.6 \mathrm{MHz}\right) \square-35.1 \mathrm{ppm} .{ }^{19} \mathrm{~F}$ NMR $\left(\mathrm{C}_{6} \mathrm{D}_{6}, 282 \mathrm{MHz}\right) \square-$ 14.1, -14.8 ppm. FT-IR (Nujol) 3277 (s), 1450 (s), 1418 (w), 1400 (w), 1328 (w), 1204 (s), 1192 (s), 1187 (s), 1139 (s), 1060 (m), 1021 (m), 993 (m), 940 (s) $\mathrm{cm}^{-1}$.

Preparation of phosphine sulfonamide 3: Lithiated phosphine sulfonamide 1d was generated as described above. To the solution of $\mathbf{1 d}$ was added acetic acid (290 $\square \mathrm{L}, 5.0 \mathrm{mmol}, 1.0$ equiv), immediately forming a precipitate. A workup analogous to that for $\mathbf{2 d}$ afforded pure phosphine sulfonamide $\mathbf{3}$ as a mixture of diastereomers at phosphorous $(1.53 \mathrm{~g}, 4.4 \mathrm{mmol}, 88 \%, \mathrm{dr}=1.3: 1.0)$ which were identified by ${ }^{1} \mathrm{H}$ and ${ }^{31} \mathrm{P}$ NMR spectroscopy and used without further purification. ${ }^{1} \mathrm{H}$ NMR $\left(\mathrm{C}_{6} \mathrm{D}_{6}, 400 \mathrm{MHz}\right) \square 4.56(\mathrm{~d}$, $J=10 \mathrm{~Hz}, 1 \mathrm{H}, \mathrm{NH}), 4.27(\mathrm{~d}, J=10 \mathrm{~Hz}, 1 \mathrm{H}, \mathrm{NH}), 3.40$ (m, 2H, CH-both diastereomers), 3.05 (dddd, ${ }^{1} J_{\mathrm{P}-\mathrm{H}}$ $=197 \mathrm{~Hz}$ (other couplings not resolved), $1 \mathrm{H}, \mathrm{PH}), 2.89$ (dddd, ${ }^{1} J_{\mathrm{P}-\mathrm{H}}=199 \mathrm{~Hz}$ (other couplings not resolved), 1H, PH), 1.85 (m, 1H, $\mathbf{C H}_{2}$ ), 0.90-1.72 (m, 22H, $\mathrm{C}_{6} \mathbf{H}_{11}$ and $\mathrm{CH}_{2}$-both diastereomers), 0.62 (s, 9H, C $\left.(\mathrm{CH})_{3}\right), 0.62\left(\mathrm{~s}, 9 \mathrm{H}, \mathrm{C}\left(\mathrm{CH}_{3}\right)_{3}\right) \mathrm{ppm} .{ }^{31} \mathrm{P}\left\{{ }^{1} \mathrm{H}\right\} \mathrm{NMR}\left(\mathrm{C}_{6} \mathrm{D}_{6}, 121.6 \mathrm{MHz}\right) \square-58.9$ (br s) ppm.

Preparation of $(S)-N$-[3,5-bis(trifluoromethyl)benzenesulfonyl]-2-tert-butylaziridine: To a cooled $\left(-10^{\circ} \mathrm{C}\right)$ solution of $(S)$-tert-leucinol (1.00 g, $8.5 \mathrm{mmol}, 1.0$ equiv), $\mathrm{Et}_{3} \mathrm{~N}$ (1.8 mL, $13.0 \mathrm{mmol}, 1.5$ equiv) and DMAP (0.095 g, $0.85 \mathrm{mmol}, 0.10$ equiv) in $\mathrm{CH}_{2} \mathrm{Cl}_{2}(10 \mathrm{~mL})$ was added a solution of 3,5bis(trifluoromethyl)benzenesulfonyl chloride $\left(2.93 \mathrm{~g}, 9.4 \mathrm{mmol}, 1.1\right.$ equiv) in $\mathrm{CH}_{2} \mathrm{Cl}_{2}$. The reaction mixture was allowed to warm to ambient temperature slowly overnight (12 h). The reaction mixture was re-cooled to $-10{ }^{\circ} \mathrm{C}$, and methanesulfonyl chloride $\left(0.73 \mathrm{~mL}, 9.4 \mathrm{mmol}, 1.1\right.$ equiv) and $\mathrm{Et}_{3} \mathrm{~N}(1.8 \mathrm{~mL}, 13.0$ mmol, 1.5 equiv) were added sequentially. The reaction mixture was again allowed to warm slowly to ambient temperature $(12 \mathrm{~h})$. The reaction mixture was diluted with $\mathrm{CH}_{2} \mathrm{Cl}_{2}(100 \mathrm{~mL})$ and washed with $\mathrm{H}_{2} \mathrm{O}(2 \times 50 \mathrm{~mL})$. The aqueous washings were extracted with $\mathrm{CH}_{2} \mathrm{Cl}_{2}(2 \times 25 \mathrm{~mL})$. The combined organic layers were stirred with $\mathrm{K}_{2} \mathrm{CO}_{3}(\mathrm{aq})$ for $1.5 \mathrm{~h}$. The organic layer was separated, dried $\left(\mathrm{MgSO}_{4}\right)$, filtered, and concentrated to afford the unpurified aziridine as a pale peach oil. Chromatography $\left(\mathrm{SiO}_{2}, 5 \% \mathrm{Et}_{2} \mathrm{O}\right.$, 95\% pentane) afforded the pure aziridine as a white crystalline solid (2.05 g, $5.4 \mathrm{mmol}, 64 \%)$. ${ }^{1} \mathrm{H}$ NMR $\left(\mathrm{CDCl}_{3}, 300 \mathrm{MHz}\right) \square 8.40$ (s, 2H,o-ArH), 8.12 (s, 1H, p-ArH), 2.78 (dd, J = $\left.7 \mathrm{~Hz}, 5 \mathrm{~Hz}, 1 \mathrm{H}, \mathrm{CH}\right), 2.66$ $\left(\mathrm{d}, J=7 \mathrm{~Hz}, \mathrm{CH}_{2}\right), 2.30\left(\mathrm{~d}, J=5 \mathrm{~Hz}, \mathrm{CH}_{2}\right), 0.85\left(\mathrm{~s}, 9 \mathrm{H}, \mathrm{C}\left(\mathrm{CH}_{3}\right)_{3}\right) \mathrm{ppm} .{ }^{13} \mathrm{C}\left\{{ }^{1} \mathrm{H}\right\} \mathrm{NMR}\left(\mathrm{CDCl}_{3}, 75 \mathrm{MHz}\right)$ 
141.6, $133.1(\mathrm{q}, J=34 \mathrm{~Hz}), 128.5,127.1,122.5(\mathrm{q}, J=272 \mathrm{~Hz}), 50.1,31.8,30.4,26.2 \mathrm{ppm} .{ }^{19} \mathrm{~F} \mathrm{NMR}$ $\left(\mathrm{CDCl}_{3}, 282 \mathrm{MHz}\right) \square-0.23$ ppm. FT-IR (thin film) 3095 (w), 2970 (m), 2875 (w), 1625 (m), 1466 (m), 1403 (w), 1360 (m), 1336 (s), 1314 (m), 1281 (s), 1234 (m), 1164 (s), 1137 (s), 984 (m), 935 (m), 903 (m), $860(\mathrm{~m}), 843(\mathrm{~m}), 823(\mathrm{w}), 736(\mathrm{~m}), 706(\mathrm{w}), 696(\mathrm{~m}), 681(\mathrm{~m}), 623(\mathrm{~m}) \mathrm{cm}^{-1}$.

Preparation and separation of diastereomeric phosphine-borane complexes $4 \mathrm{a}$ and $4 \mathrm{~b}$ : In an inert atmosphere glovebox, a $50 \mathrm{~mL}$ resealable glass ampoule (w/teflon stopcock) was charged with phosphine sulfonamide 3 (0.847 g, $2.43 \mathrm{mmol})$, (S)- $N$-[3,5-bis(trifluoromethyl)benzenesulfonyl]-2-tertbutylaziridine (0.913 g, $2.43 \mathrm{mmol})$, and 2,2,2-trifluoroethanol (15 mL). The reaction vessel was sealed, removed from glovebox, and placed in a $70{ }^{\circ} \mathrm{C}$ controlled-temperature bath for $48 \mathrm{~h}$. After this period volatile materials were removed in vacuo; while maintaining an inert atmosphere, the resulting white solid was taken up in ethyl ether $(10 \mathrm{~mL})$ to give a clear, nearly colorless solution, which was cooled to $-10{ }^{\circ} \mathrm{C}$. A solution of $\mathrm{BH}_{3} \cdot \mathrm{DMS}(7.0 \mathrm{~mL}$ of $2.0 \mathrm{M}$ solution in THF, $13.8 \mathrm{mmol})$ was added dropwise. Upon completion of borane addition, the reaction mixture was permitted to warm to ambient temperature over 1 h. The reaction mixture was then re-cooled $\left(-10^{\circ} \mathrm{C}\right)$ and quenched by careful addition of distilled $\mathrm{H}_{2} \mathrm{O}(3$ $\mathrm{mL}$ ). After warming to room temperature with rapid stirring over $1.5 \mathrm{~h}$, the reaction mixture was diluted with ether $(50 \mathrm{~mL})$ and filtered through a short pad of $\mathrm{MgSO}_{4} / \mathrm{silica}$ gel on a coarse fritted funnel. The $\mathrm{MgSO}_{4} /$ silica gel pad was washed with an additional 3 x $50 \mathrm{~mL}$ of ether. The combined ethereal fractions were dried $\left(\mathrm{MgSO}_{4}\right)$, filtered through Celite, and concentrated to afford the crude mixture of $\mathbf{4 a} / \mathbf{4 b}$ as a slightly cloudy oil. ${ }^{1} \mathrm{H}$ and ${ }^{19} \mathrm{~F}$ NMR spectroscopic analysis of the crude material revealed the diastereomers were present in the ratio of $(S, R, S):(S, S, S)=1.5: 1.0$. The diastereomers were separated by flash silica gel chromatography (4:1 pentane:ether) to afford diastereopure $\mathbf{4 a}((S, R, S)$ configuration) $(0.768 \mathrm{~g})$ and diastereopure $\mathbf{4 b}((S, S, S)$ configuration) $(0.479 \mathrm{~g})$ as white solids. Re-chromatography of the $\sim 0.200 \mathrm{~g}$ of mixed fractions afforded an additional $0.105 \mathrm{~g}$ of $\mathbf{4 a}$ and $0.069 \mathrm{~g}$ of $\mathbf{4 b}$ as white solids. Total yield for both diastereomers: $1.421 \mathrm{~g}(1.92 \mathrm{mmol}, 79 \%) .4 a:{ }^{1} \mathrm{H} \mathrm{NMR}\left(\mathrm{CDCl}_{3}, 300 \mathrm{MHz}\right) \square 8.24$ (s, $\left.2 \mathrm{H}, o-\mathrm{C}_{6} \mathbf{H}_{3}\right), 8.04\left(\mathrm{~s}, 1 \mathrm{H}, p-\mathrm{C}_{6} \mathbf{H}_{3}\right), 7.00(\mathrm{~d}, J=10 \mathrm{~Hz}, 1 \mathrm{H}, \mathrm{NH}), 5.72(\mathrm{~d}, J=10 \mathrm{~Hz}, 1 \mathrm{H}, \mathrm{NH}), 4.02(\mathrm{~m}$, 1H, NCH), 3.53 (m, 1H, NCH), 2.70 (m, 1H, PCH), 1.98-2.40 (m, 4H, both PCH $), 1.20-1.87$ (m, $10 \mathrm{H}$, $\left.\left(\mathrm{CH}_{2}\right)_{5}\right), 1.02\left(\mathrm{~s}, 9 \mathrm{H},\left(\mathrm{CH}_{3}\right)_{3} \mathrm{C}\right), 0.66\left(\mathrm{~s}, 9 \mathrm{H},\left(\mathrm{CH}_{3}\right)_{3} \mathrm{C}\right) \mathrm{ppm}$. (Presumably, the three $\mathrm{BH}_{3}$ protons are 
broadened into the baseline due to coupling with spin-active ${ }^{10} \mathrm{~B},{ }^{11} \mathrm{~B}$, and ${ }^{31} \mathrm{P}$ nuclei.) ${ }^{13} \mathrm{C}\left\{{ }^{1} \mathrm{H}\right\} \mathrm{NMR}$ $\left(\mathrm{CDCl}_{3}, 75 \mathrm{MHz}\right), \square 143.8,133.2(\mathrm{q}, J=34 \mathrm{~Hz}), 127.3,126.5,122.6(\mathrm{q}, J=272 \mathrm{~Hz}), 117.0(\mathrm{q}, J=319$ Hz), $61.2(\mathrm{~d}, J=10 \mathrm{~Hz}), 60.1,37.1(\mathrm{~d}, J=9 \mathrm{~Hz}), 36.9$ (d, $J=7 \mathrm{~Hz}), 29.8$ (d, $J=34 \mathrm{~Hz}), 26.8,26.6,26.5$, 26.3, 26.1, 25.9, 24.1 (d, $J=20 \mathrm{~Hz}), 23.8(\mathrm{~d}, J=28 \mathrm{~Hz}) \mathrm{ppm} .{ }^{31} \mathrm{P}\left\{{ }^{1} \mathrm{H}\right\} \mathrm{NMR}\left(\mathrm{CDCl}_{3}, 121.6 \mathrm{MHz}\right) \square 20.2$ ppm. ${ }^{19} \mathrm{~F}\left(\mathrm{CDCl}_{3}, 282 \mathrm{MHz}\right) \square-0.27$ (s, 6F, $\left.\operatorname{Ar}\left(\mathrm{CF}_{3}\right)_{2}\right),-12.02$ (s, 3F, $\left.\mathrm{SO}_{2} \mathrm{CF}_{3}\right)$ ppm. FT-IR (thin film) 3309 (s), 2967 (m), 2938 (m), 2859 (m), 2377 (s), $1626(\mathrm{w}), 1474(\mathrm{~m}), 1450$ (m), 1362 (s), $1316(\mathrm{w}), 1281$ (s), 1188 (s), 1151 (s), $1111(\mathrm{~m}), 1059$ (m), 1023 (m), 926 (w), 906 (m), $845(\mathrm{w}), 833$ (w), $776(\mathrm{w}), 669$ (m), $682(\mathrm{~m}), 634(\mathrm{~m}), 620(\mathrm{~m}), 594(\mathrm{~m}), 570(\mathrm{~m}) \mathrm{cm}^{-1} . \mathrm{MS}(\mathrm{FAB}+) \mathrm{m} / z=735.50\left(\mathrm{M}^{+}-\mathrm{H}\right) .4 \mathrm{~b}:{ }^{1} \mathrm{H}$ NMR $\left(\mathrm{CDCl}_{3}, 300 \mathrm{MHz}\right) \square 8.22\left(\mathrm{~s}, 2 \mathrm{H}, o-\mathrm{C}_{6} \mathbf{H}_{3}\right), 8.07\left(\mathrm{~s}, 1 \mathrm{H}, p-\mathrm{C}_{6} \mathbf{H}_{3}\right), 6.27(\mathrm{~d}, J=10 \mathrm{~Hz}, 1 \mathrm{H}, \mathrm{NH}), 6.22(\mathrm{~d}, J=$ $10 \mathrm{~Hz}, 1 \mathrm{H}, \mathrm{NH}), 4.03$ (m, 1H, NCH), 3.53 (m, 1H, NCH), 2.60 (m, 1H, PCH), 2.18 (m, 4H, both PCH $)$, 1.20-1.88 (m, 10H, $\left.\left(\mathrm{CH}_{2}\right)_{5}\right), 1.00\left(\mathrm{~s}, 9 \mathrm{H},\left(\mathrm{CH}_{3}\right)_{3} \mathrm{C}\right), 0.67\left(\mathrm{~s}, 9 \mathrm{H},\left(\mathrm{CH}_{3}\right)_{3} \mathrm{C}\right) \mathrm{ppm}$. (Presumably, the three $\mathrm{BH}_{3}$ protons are broadened into the baseline due to coupling with spin-active ${ }^{10} \mathrm{~B},{ }^{11} \mathrm{~B}$, and ${ }^{31} \mathrm{P}$ nuclei.) ${ }^{13} \mathrm{C}\left\{{ }^{1} \mathrm{H}\right\} \operatorname{NMR}\left(\mathrm{CDCl}_{3}, 75 \mathrm{MHz}\right) \square 143.6,132.9(\mathrm{q}, J=35 \mathrm{~Hz}), 126.8,126.3,122.3(\mathrm{q}, J=272 \mathrm{~Hz}), 119.0$ $(\mathrm{q}, J=319 \mathrm{~Hz}), 60.6,60.0(\mathrm{~d}, J=10 \mathrm{~Hz}), 36.8(\mathrm{~d}, J=7 \mathrm{~Hz}), 36.5(\mathrm{~d}, J=9 \mathrm{~Hz}), 29.9$ (d, $J=35 \mathrm{~Hz}), 26.6$, 26.2, 25.7, 25.6, 23.6 (d, $J=25 \mathrm{~Hz}), 22.2(\mathrm{~d}, J=28 \mathrm{~Hz}) \mathrm{ppm} .{ }^{31} \mathrm{P}\left\{{ }^{1} \mathrm{H}\right\} \mathrm{NMR}\left(\mathrm{CDCl}_{3}, 121.6 \mathrm{MHz}\right) \square 20.4$ ppm. ${ }^{19} \mathrm{~F} \mathrm{NMR}\left(\mathrm{CDCl}_{3}, 282 \mathrm{MHz}\right) \square-0.25\left(\mathrm{~s}, 6 \mathrm{~F}, \operatorname{Ar}\left(\mathrm{CF}_{3}\right)_{2}\right),-11.67$ (s, 3F, SOCF $)$ ppm. FT-IR (thin film) 3292 (s), 2938 (s), 2380 (m), 1450 (m), 1361 (s), 1281 (s), 1189 (s), 1151 (s), 1061 (m), 1021 (m), 906 (w), $682(\mathrm{~m}) \mathrm{cm}^{-1}$. MS (FAB+) $m / z=735.50\left(\mathrm{M}^{+}-\mathrm{H}\right)$.

Preparation of ligand 5a: A $50 \mathrm{~mL}$ resealable glass ampoule (w/Teflon stopcock) was charged with 4a (0.215 g, $0.292 \mathrm{mmol})$, DABCO (0.098 g, $0.975 \mathrm{mmol})$ and dry, degassed benzene (4 mL). The ampoule was sealed and placed in a $55^{\circ} \mathrm{C}$ controlled-temperature bath for $22 \mathrm{~h}$. Upon cooling to room temperature, the homogeneous benzene solution was frozen and the solvent lyophilized, affording a white solid. In the glovebox, this material was taken up in $\mathrm{Et}_{2} \mathrm{O}(\sim 3 \mathrm{~mL})$ and filtered through a short pad of silica gel on a fritted funnel. The silica pad was washed with several additional small portions of ether to ensure complete elution of the product. The combined ethereal washings were concentrated to give a white foamy, sticky solid. This was taken up in benzene, the resulting solution frozen, and the solvent lyophilized to afford pure 5a as a white powder $(0.177 \mathrm{~g}, 0.245 \mathrm{mmol}, 84 \%) .{ }^{1} \mathrm{H} \mathrm{NMR}\left(\mathrm{CDCl}_{3}, 400 \mathrm{MHz}\right)$ 
$8.30\left(\mathrm{~s}, 2 \mathrm{H}, o-\mathrm{C}_{6} \mathbf{H}_{3}\right), 8.00\left(\mathrm{~s}, 1 \mathrm{H}, p-\mathrm{C}_{6} \mathbf{H}_{3}\right), 5.82(\mathrm{br} \mathrm{s}, 1 \mathrm{H}, \mathrm{NH}), 5.38(\mathrm{~d}, J=9 \mathrm{~Hz}, 1 \mathrm{H}, \mathrm{NH}), 3.47$ (m, 1H, NCH), 3.37 (m, 1H, NCH), 1.65-2.10 and 1.08-1.42 (m, 15H, $\mathrm{C}_{6} \mathbf{H}_{11}$ and both PCH $), 0.98(\mathrm{~s}, 9 \mathrm{H}$, $\left.\left(\mathrm{CH}_{3}\right)_{3} \mathrm{C}\right), 0.86\left(\mathrm{~s}, 9 \mathrm{H},\left(\mathrm{CH}_{3}\right)_{3} \mathrm{C}\right) \mathrm{ppm} .{ }^{13} \mathrm{C}\left\{{ }^{1} \mathrm{H}\right\} \mathrm{NMR}\left(\mathrm{CDCl}_{3}, 75 \mathrm{MHz}\right) \square 144.8,132.4(\mathrm{q}, J=34 \mathrm{~Hz})$, 127.3, 125.6, $122.6(\mathrm{q}, J=271 \mathrm{~Hz}), 119.1(\mathrm{q}, J=319 \mathrm{~Hz}), 64.1(\mathrm{~d}, J=20 \mathrm{~Hz}), 63.1(\mathrm{~d}, J=10 \mathrm{~Hz}), 36.9$ $(\mathrm{d}, J=11 \mathrm{~Hz}), 36.1$ (d, $J=7 \mathrm{~Hz}), 35.7,27.4$ (d, $J=4 \mathrm{~Hz}), 26.5 \mathrm{ppm}$. Resonances corresponding to the seven methylene carbons of 5a appear between 26.5-30.0 ppm, but were insufficiently resolved to determine precise chemical shifts and coupling constants. ${ }^{31} \mathrm{P}\left\{{ }^{1} \mathrm{H}\right\} \mathrm{NMR}\left(\mathrm{CDCl}_{3}, 282 \mathrm{MHz}\right) \square-35.3 \mathrm{ppm}$. ${ }^{19} \mathrm{~F} \mathrm{NMR}\left(\mathrm{CDCl}_{3}, 282 \mathrm{MHz}\right) \square-0.26\left(\mathrm{~s}, 6 \mathrm{~F}, \mathrm{Ar}\left(\mathrm{CF}_{3}\right)_{2}\right),-13.57$ (s, 3F, $\left.\mathrm{SO}_{2} \mathrm{CF}_{3}\right)$ ppm. FT-IR (Nujol) 3381 (m), 3278 (s), $1626(\mathrm{w}), 1406(\mathrm{w}), 1357$ (m), 1332 (m), $1314(\mathrm{w}), 1279(\mathrm{~s}), 1266(\mathrm{~m}), 1203(\mathrm{~s}), 1175(\mathrm{~m})$, 1154 (s), 1111 (m), 1068 (m), 1022 (m), 938 (w), 913 (w), 889 (w), $844(w), 811(w), 768(w) \mathrm{cm}^{-1}$. MS $(\mathrm{FAB}+) m / z=723.50\left(\mathbf{M}^{+}+\mathrm{H}\right)$.

Preparation of ligand 5b: The preparation of ligand $\mathbf{5 b}$ was entirely analogous to that of $\mathbf{5 a}$, affording pure $\mathbf{5 b}$ in $76 \%$ yield. ${ }^{1} \mathrm{H} \mathrm{NMR}\left(\mathrm{CDCl}_{3}, 400 \mathrm{MHz}\right) \square 8.26\left(\mathrm{~s}, 2 \mathrm{H}, o-\mathrm{C}_{6} \mathbf{H}_{3}\right), 8.03\left(\mathrm{~s}, 1 \mathrm{H}, p-\mathrm{C}_{6} \mathbf{H}_{3}\right)$, $6.39(\mathrm{~d}, J=10 \mathrm{~Hz}, 1 \mathrm{H}, \mathrm{NH}), 6.08(\mathrm{dd}, J=7 \mathrm{~Hz}, 3 \mathrm{~Hz}, 1 \mathrm{H}, \mathrm{NH}), 3.57$ (m, 1H, NCH), 3.36 (m, 1H, NCH), $2.12\left(\mathrm{~m}, 1 \mathrm{H}, \mathrm{PCH}_{2}\right), 2.03$ (m, 1H, PCH $), 1.64-1.81,1.50-1.60,1.25-1.35\left(\mathrm{~m}, 13 \mathrm{H}, \mathrm{C}_{6} \mathbf{H}_{11}\right.$ and $\mathrm{PCH}_{2}$ ), $1.00\left(\mathrm{~s}, 9 \mathrm{H}, \mathrm{C}(\mathrm{CH})_{3}, 0.74\left(\mathrm{~s}, 9 \mathrm{H}, \mathrm{C}(\mathrm{CH})_{3}\right) \mathrm{ppm} .{ }^{13} \mathrm{C}\left\{{ }^{1} \mathrm{H}\right\} \mathrm{NMR}\left(75 \mathrm{MHz}, \mathrm{CDCl}_{3}\right) \square 143.6,132.8(\mathrm{q}, J=34\right.$ $\mathrm{Hz}), 127.0,126.0,122.4(\mathrm{q}, J=272 \mathrm{~Hz}), 119.5(\mathrm{q}, J=318 \mathrm{~Hz}), 66.0(\mathrm{~d}, J=22 \mathrm{~Hz}), 61.8(\mathrm{~d}, J=10 \mathrm{~Hz})$, $31.2(\mathrm{~d}, J=17 \mathrm{~Hz}), 36.0,35.6(\mathrm{~d}, J=7 \mathrm{~Hz}), 31.2(\mathrm{~d}, J=17 \mathrm{~Hz}), 30.1(\mathrm{~d}, J=13 \mathrm{~Hz}), 29.0(\mathrm{~d}, J=11 \mathrm{~Hz})$, $27.5(\mathrm{~d}, J=5 \mathrm{~Hz}), 26.5 \mathrm{ppm}$. Resonances corresponding to the methylene carbons of the $\mathrm{C}_{6} \mathrm{H}_{11}$ ring appear between 26.3-27.2 ppm, but were insufficiently resolved to determine precise chemical shifts and coupling constants. ${ }^{31} \mathrm{P}$ NMR $\left(\mathrm{CDCl}_{3}, 282 \mathrm{MHz}\right) \square-35.0 \mathrm{ppm} .{ }^{19} \mathrm{~F} \mathrm{NMR}\left(\mathrm{CDCl}_{3}, 282 \mathrm{MHz}\right) \square-0.37$ (s, 6F, $\left.\operatorname{Ar}\left(\mathrm{CF}_{3}\right)_{2}\right),-14.36\left(\mathrm{~S}, 3 \mathrm{~F}, \mathrm{SO}_{2} \mathrm{CF}_{3}\right)$ ppm. FT-IR (Nujol) 3280 (s), 1626 (w), 1370 (s), 1279 (s), 1188 (s), 1150 (s), $1112(\mathrm{~m}), 1066(\mathrm{~m}), 1018(\mathrm{~m}), 994(\mathrm{~m}), 936(\mathrm{~m}), 905(\mathrm{~m}), 844(\mathrm{w}), 822(\mathrm{w}), 776(\mathrm{w}), 723(\mathrm{w})$, $700(\mathrm{~m}), 682(\mathrm{~m}), 634(\mathrm{~m}), 615(\mathrm{~m}) \mathrm{cm}^{-1} . \mathrm{MS}(\mathrm{FAB}+) \mathrm{m} / z=723.50\left(\mathrm{M}^{+}+\mathrm{H}\right)$.

Preparation of ligand 6: A $25 \mathrm{~mL}$ resealable glass ampoule (w/ Teflon stopcock) was charged with cyclohexylphosphine (22 $\square \mathrm{L}, 0.167 \mathrm{mmol}, 1.0$ equiv), (S)- $N$-[3,5-bis(trifluoromethyl)benzenesulfonyl]-2tert-butylaziridine $\left(0.125 \mathrm{~g}, 0.333 \mathrm{mmol}, 2.0\right.$ equiv) and $\mathrm{CF}_{3} \mathrm{CH}_{2} \mathrm{OH}(1 \mathrm{~mL})$. The ampoule was sealed and 
placed in an $80^{\circ} \mathrm{C}$ controlled-temperature bath for $60 \mathrm{~h}$. Upon cooling to ambient temperature, volatile materials were removed in vacuo. In the glovebox, the resulting white foamy residue was taken up in $\mathrm{CH}_{2} \mathrm{Cl}_{2}(1 \mathrm{~mL})$ and filtered through a short plug of silica gel. The silica plug was eluted with an additional $20 \mathrm{~mL}$ of $\mathrm{CH}_{2} \mathrm{Cl}_{2}$ to ensure complete elution of the product. Concentration of the resulting clear, colorless solution afforded pure 6 as a white solid $(0.115 \mathrm{~g}, 0.132 \mathrm{mmol}, 79 \%) .{ }^{1} \mathrm{H} \mathrm{NMR}\left(300 \mathrm{MHz}, \mathrm{CDCl}_{3}\right) \square 8.39$ $\left(\mathrm{s}, 2 \mathrm{H}, o-\mathrm{C}_{6} \mathbf{H}_{3}\right), 8.30\left(\mathrm{~s}, 2 \mathrm{H}, o-\mathrm{C}_{6} \mathbf{H}_{3}\right), 8.06\left(\mathrm{~s}, 1 \mathrm{H}, p-\mathrm{C}_{6} \mathbf{H}_{3}\right), 8.01\left(\mathrm{~s}, 1 \mathrm{H}, p-\mathrm{C}_{6} \mathbf{H}_{3}\right), 5.93(\mathrm{~d}, J=9 \mathrm{~Hz}, 1 \mathrm{H}$, NH), $5.62(\mathrm{dd}, J=10 \mathrm{~Hz}, 3 \mathrm{~Hz}, 1 \mathrm{H}, \mathrm{NH}), 3.40$ (m, 2H, both of NCH), 1.00-2.05 (m, 15H, $\mathrm{C}_{6} \mathbf{H}_{11}$ and both of $\left.\mathrm{PCH}_{2}\right), 0.89$ (s, 9H, C(CH$\left.)_{3}\right), 0.66\left(\mathrm{~s}, 9 \mathrm{H}, \mathrm{C}\left(\mathrm{CH}_{3}\right)_{3}\right) \mathrm{ppm} .{ }^{13} \mathrm{C}\left\{{ }^{1} \mathrm{H}\right\} \mathrm{NMR}\left(\mathrm{CDCl}_{3}, 75 \mathrm{MHz}\right) \square$ 145.2, 143.8, $132.8(\mathrm{q}, J=43 \mathrm{~Hz}), 132.4(\mathrm{q}, J=43 \mathrm{~Hz}), 127.4,127.0,126.0,125.4,122.7(\mathrm{q}, J=272 \mathrm{~Hz}), 122.4$ $(\mathrm{q}, J=272 \mathrm{~Hz}), 64.3(\mathrm{~d}, J=19 \mathrm{~Hz}), 61.6(\mathrm{~d}, J=9 \mathrm{~Hz}), 36.9(\mathrm{~d}, J=11 \mathrm{~Hz}), 36.2(\mathrm{~d}, J=6 \mathrm{~Hz}), 36.0,27.2$ $(\mathrm{d}, J=3 \mathrm{~Hz}), 26.6 \mathrm{ppm}$. Resonances for the seven methylene carbons of $\mathbf{6}$ appear between 29.3-29.8 and 26.2-27.2 ppm, but are insufficiently resolved to determine precise chemical shifts and coupling constants. ${ }^{31} \mathrm{P}\left\{{ }^{1} \mathrm{H}\right\} \mathrm{NMR}\left(\mathrm{CDCl}_{3}, 121.6 \mathrm{MHz}\right) \square-35.5 \mathrm{ppm} .{ }^{19} \mathrm{~F} \mathrm{NMR}\left(\mathrm{CDCl}_{3}, 282 \mathrm{MHz}\right), \square-0.24(\mathrm{~s}, 6 \mathrm{~F}$, $\left.\operatorname{Ar}\left(\mathrm{CF}_{3}\right)_{2}\right)$-0.43 (s, 6F, Ar(CF $\left.)_{2}\right)$ ppm. FT-IR (Nujol) 3266 (s), 1626 (w), 1370 (s), 1317 (m), 1279 (s), 1150 (s), 1113 (s), 1068 (m), 1022 (m), 934 (m), 906 (m), 844 (m), $830(\mathrm{w}), 804$ (w), $771(\mathrm{w}), 722$ (w), $700(\mathrm{~m}), 682(\mathrm{~s}), 632(\mathrm{~s}), 593(\mathrm{~s}) \mathrm{cm}^{-1}$. MS (FAB+) $m / z=866.99(\mathrm{M}+)$.

Representative procedure for conjugate addition reactions with $\mathrm{Cu}(\mathrm{OTf})_{2} / 5 \mathrm{a} / \mathrm{Et}_{2} \mathrm{Zn}$ : In a glovebox, a $10 \mathrm{~mL}$ round-bottom flask was charged with $\mathrm{Cu}(\mathrm{OTf})_{2}(0.0037 \mathrm{~g}, 0.0103 \mathrm{mmol})$ and 5a $(0.0111 \mathrm{~g}, 0.0154 \mathrm{mmol})$. The flask was sealed with a septum, removed from the glovebox, and maintained under an oil-bubbler pressure of $\mathrm{N}_{2} \cdot \mathrm{Et}_{2} \mathrm{O}(2 \mathrm{~mL})$ was added, and the resulting mixture was stirred at room temperature for $15 \mathrm{~min}$ to afford a clear, colorless solution. $\mathrm{Et}_{2} \mathrm{Zn}(86 \square \mathrm{L}, 0.821 \mathrm{mmol})$ was added dropwise, resulting in an immediate color change to yellow, which gradually faded over 15 min to a homogeneous pale pink solution. A solution of benzylidene acetone $(0.100 \mathrm{~g}, 0.684 \mathrm{mmol})$ in $\mathrm{Et}_{2} \mathrm{O}(1.5 \mathrm{~mL})$ was then added dropwise via syringe (note: other enones were added neat to the $\mathrm{Cu}(\mathrm{OTf})_{2} / \mathbf{5 a} / \mathrm{Et}_{2} \mathrm{Zn}$ mixture). When, after 1-1.5 h, it was observed by TLC that the enone was entirely consumed, the reaction was quenched by dropwise addition of $1 \mathrm{~N} \mathrm{HCl}$. The resulting heterogeneous mixture was diluted with $\mathrm{Et}_{2} \mathrm{O}(10 \mathrm{~mL})$, filtered through Celite, and concentrated to give a yellow oil. 
The crude material was purified on silica gel $\left(10 \% \mathrm{Et}_{2} \mathrm{O}, 90 \%\right.$ pentane) to give pure $(R)-(-)-4-$ phenylhexan-2-one $(0.101 \mathrm{mg}, 0.575 \mathrm{mmol}, 84 \%)$ as a clear pale yellow oil.

\section{Characterization and stereochemical determinations and proofs for conjugate addition} products:

(R)-(-)-4-Phenylhexan-2-one (Table 3, entry 1): Enantiomeric excess was determined by chiral GC: Astec Chiraldex G-TA column; $2.0 \mathrm{~mL} / \mathrm{min}$ He flow, $100{ }^{\circ} \mathrm{C}$. $[\square]_{\mathrm{D}}{ }^{20}=-28.7^{\circ}(\mathrm{c}=1.00$, EtOH $)$ for an $87 \%$ ee sample. This is a known compound; it has previously been established that the $(R)$ enantiomer has a negative sign of rotation. ${ }^{1}$
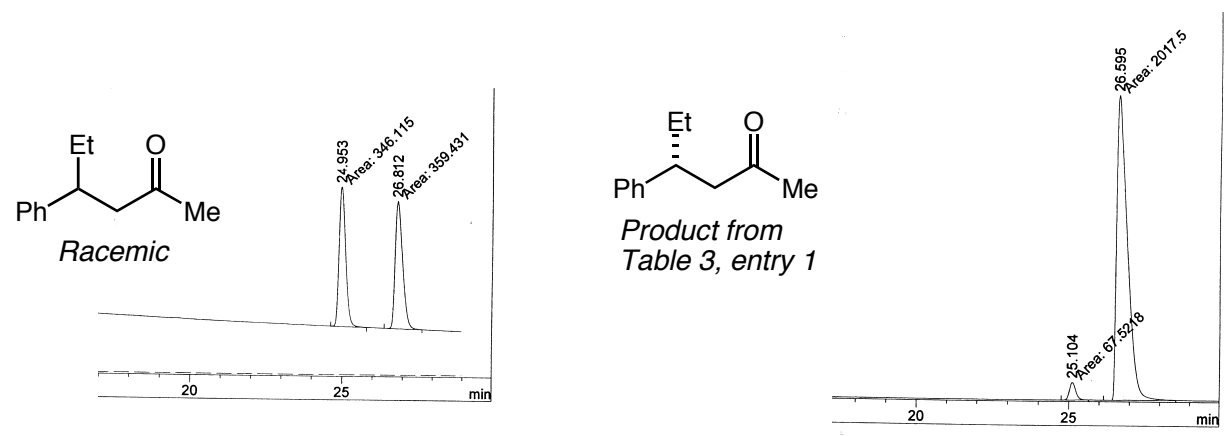

(R)-(-)-3-Methylundecan-5-one (Table 3, entry 2): Enantiomeric excess was determined by chiral GC: Astec Chiraldex G-TA column; $1.0 \mathrm{~mL} / \mathrm{min}$ He flow, $80{ }^{\circ} \mathrm{C}$. $[\square]_{\mathrm{D}}{ }^{20}=-3.5^{\circ}\left(\mathrm{c}=1.15, \mathrm{CHCl}_{3}\right)$ for an $88 \%$ ee sample. This is a known compound, ${ }^{2}$ and the absolute configuration was assigned by analogy.

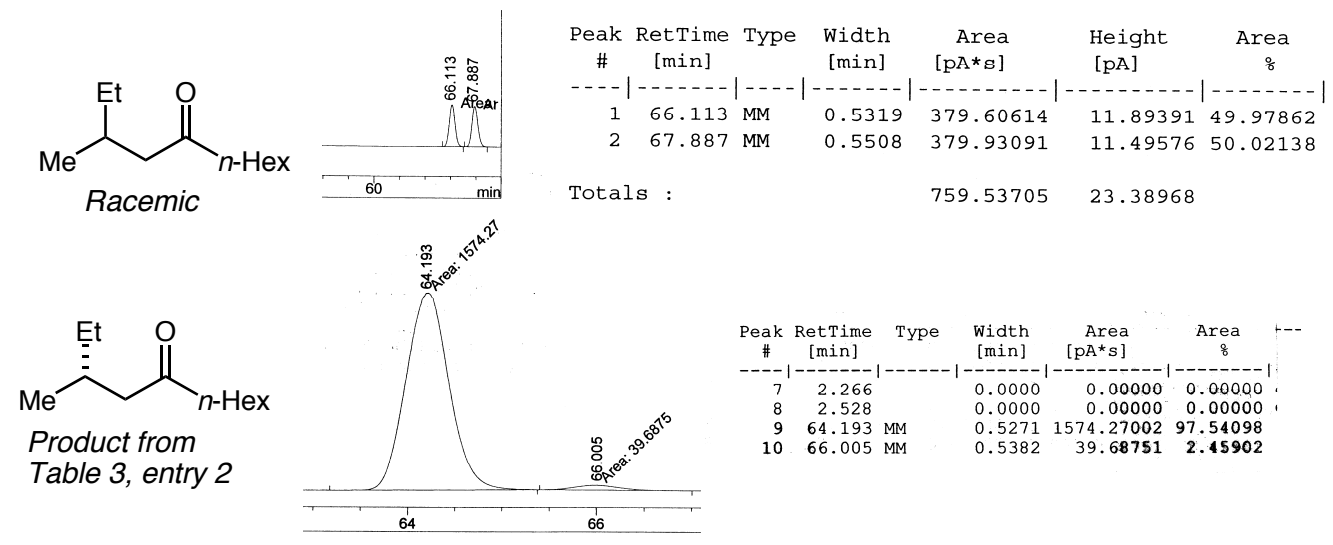

(1) Soai, K.; Yokoyama, S.; Hayasaka, T.; Ebihara, K. J. Org. Chem. 1988, 53, 4148.

(2) Mizutani, H.; Degrado, S. J.; Hoveyda, A. H. J. Am. Chem. Soc. 2002, 124, 779. 
(S)-(-)-4-Ethylnonan-2-one (Table 3, entry 3): Enantiomeric excess was determined by chiral GC: Astec Chiraldex G-TA column; $1.0 \mathrm{~mL} / \mathrm{min}$ He flow, $80{ }^{\circ} \mathrm{C}$. $[\square]_{\mathrm{D}}{ }^{20}=-2.0^{\circ}\left(\mathrm{c}=0.65, \mathrm{CHCl}_{3}\right)$ for an $84 \%$ ee sample. This is a known compound, ${ }^{2,3}$ and it has previously been established that the $(R)$ enantiomer has a positive sign of rotation. ${ }^{3}$
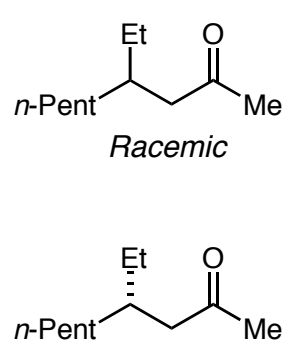

Product from Table 3, entry 2
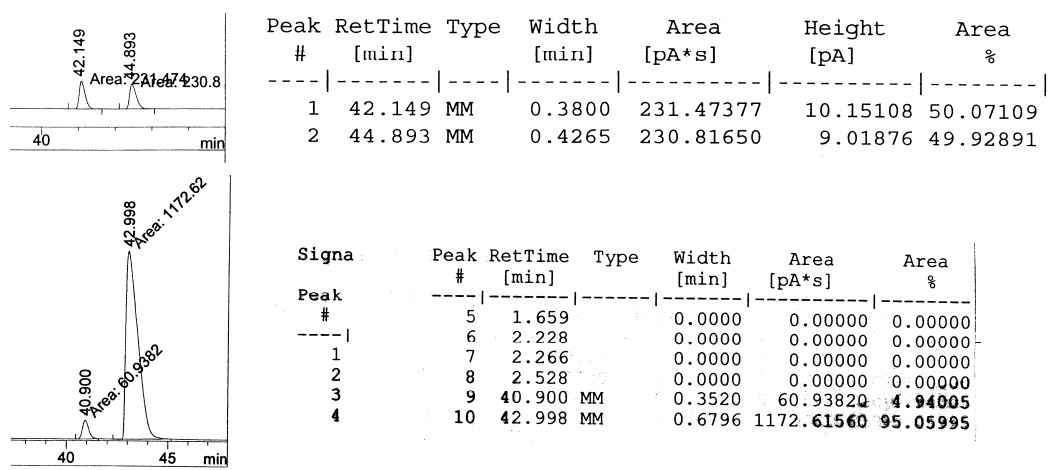
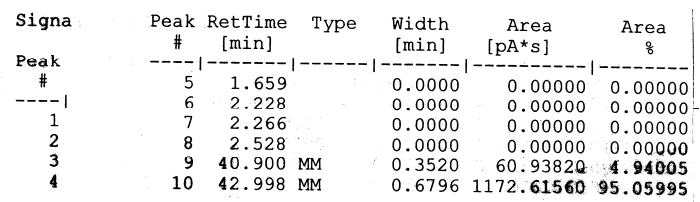

(R)-(-)-4-Ethyl-5-methylhexan-2-one (Table 3, entry 4): Enantiomeric excess was determined by chiral GC: Astec Chiraldex G-TA column; $1.0 \mathrm{~mL} / \mathrm{min} \mathrm{He}$ flow, $80{ }^{\circ} \mathrm{C}$. $[\square]_{\mathrm{D}}{ }^{20}=-3.5^{\circ}\left(\mathrm{c}=1.00, \mathrm{CHCl}_{3}\right)$ for an $85 \%$ ee sample. This is a known compound, and it has previously been established that the $(S)$ enantiomer has a positive sign of rotation. ${ }^{2}$

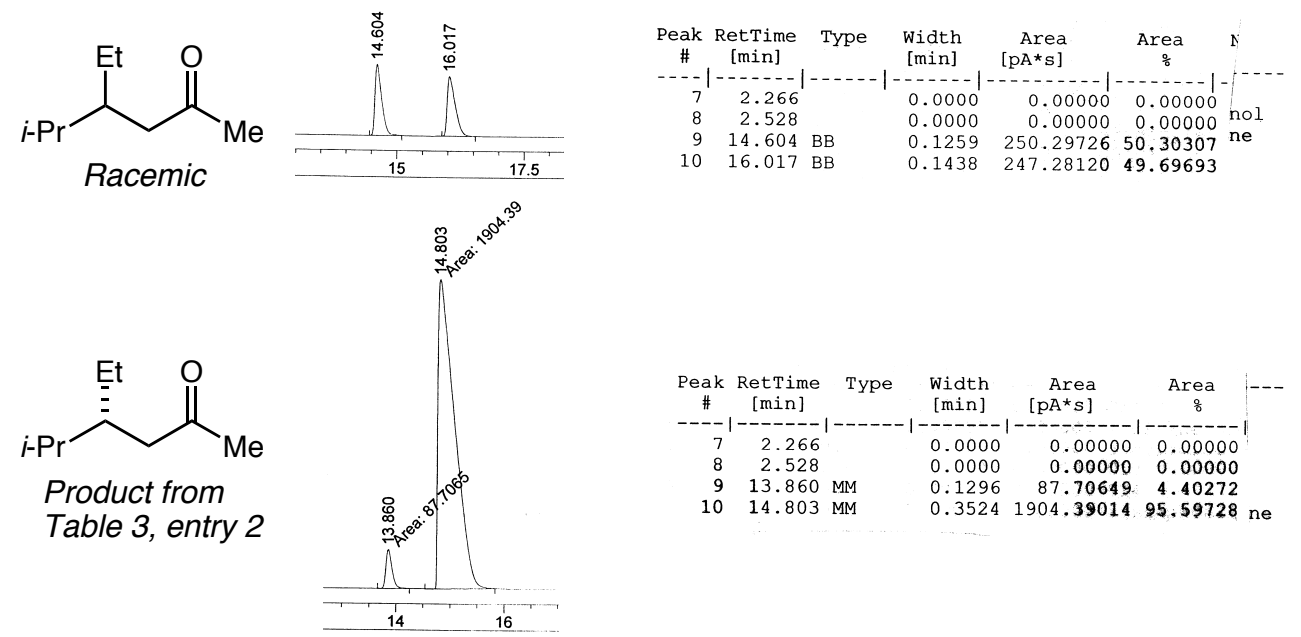

(3) Bennett, S. M. W.; Brown, S.M.; Cunningham, A.; Dennis, M. R.; Muxworthy, J. P.; Oakley, M. A.; Woodward, S. 
(R)-(+)-4-(Benzyloxymethyl)hexan-2-one (Table 3, entry 5): ${ }^{1} \mathrm{H}$ NMR $\left(\mathrm{CDCl}_{3}, 300 \mathrm{MHz}\right) \square 7.30$ (m, $\left.5 \mathrm{H}, \mathrm{C}_{6} \mathbf{H}_{5}\right), 4.46\left(\mathrm{~s}, 2 \mathrm{H}, \mathrm{PhCH}_{2} \mathrm{O}\right), 3.43\left(\mathrm{dd}, J=9 \mathrm{~Hz}, 5 \mathrm{~Hz}, 1 \mathrm{H}, \mathrm{BnOCH}_{2}\right), 3.31(\mathrm{dd}, J=9 \mathrm{~Hz}, 7 \mathrm{~Hz}, 1 \mathrm{H}$, $\left.\mathrm{BnOCH}_{2}\right), 2.54\left(\mathrm{dd}, J=16 \mathrm{~Hz}, 7 \mathrm{~Hz}, 1 \mathrm{H}, \mathrm{CH}_{2} \mathrm{C}(\mathrm{O})\right), 2.34\left(\mathrm{dd}, J=16 \mathrm{~Hz}, 6 \mathrm{~Hz}, 1 \mathrm{H}, \mathrm{CH} \mathbf{H}_{2} \mathrm{C}(\mathrm{O})\right), 2.18(\mathrm{~m}$, $1 \mathrm{H}, \mathrm{OCH}_{2} \mathrm{CH}$ ), 2.13 (s, 3H, $\mathrm{CH}_{3} \mathrm{C}(\mathrm{O}), 1.38$ (m, 2H, $\mathrm{CH}_{2} \mathrm{CH}_{3}$ ), 0.89 (t, J=8 Hz, 3H, $\left.\mathrm{CH}_{2} \mathrm{CH}_{3}\right) \mathrm{ppm}$. ${ }^{13} \mathrm{C}\left\{{ }^{1} \mathrm{H}\right\} \mathrm{NMR}\left(\mathrm{CDCl}_{3}, 75 \mathrm{MHz}\right) \square 208.9,138.4,128.3,127.5,127.4,72.9,72.5,45.9,36.2,30.4,24.3$, 11.3 ppm. FT-IR (thin film) 3065 (w), 3031 (w), 2962 (s), 2930 (s), 2861 (s), 1715 (s), 1496 (m), 1455 (s), 1410 (m), 1363 (s), 1310 (w), 1235 (w), 1206 (w), 1169 (m), 1100 (s), 1028 (m), 1002 (w), 738 (s), $699(\mathrm{~s}) \mathrm{cm}^{-1}$. MS (FAB+) $m / z=221.09\left(\mathrm{M}^{+}+\mathrm{H}\right)$.

Enantiomeric excess was determined by chiral HPLC: Chiralpak AD-H column; 99:1 hexane:isopropanol; $0.80 \mathrm{~mL} / \mathrm{min} .[\square]_{\mathrm{D}}{ }^{22}=+1.3^{\circ}\left(\mathrm{c}=1.10, \mathrm{CHCl}_{3}\right)$ for a $90 \%$ ee sample. The absolute configuration was assigned by analogy.
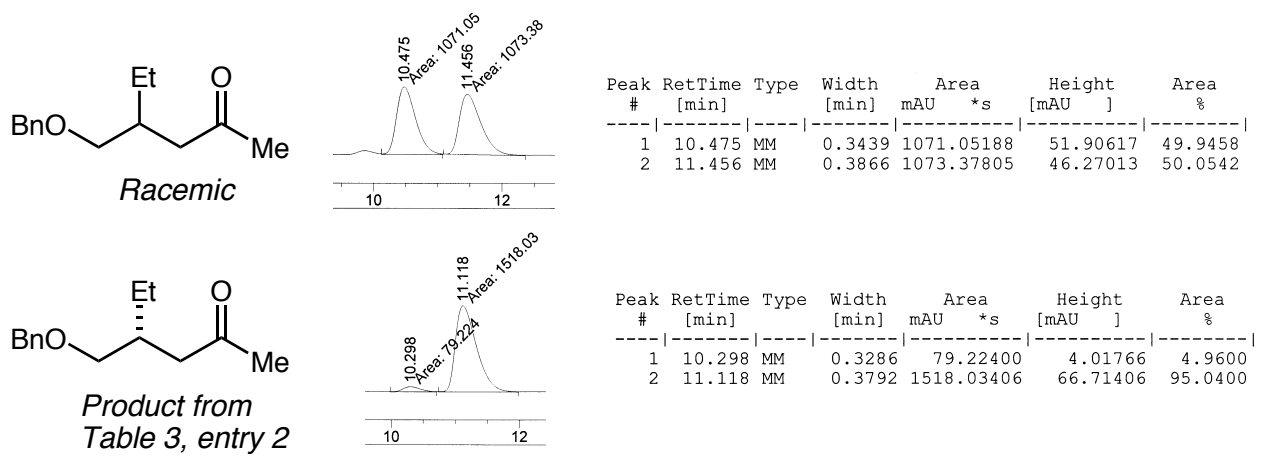

(R)-(+)-5-Benzyloxy-4-methylpentan-2-one (Scheme 5): Enantiomeric excess was determined by chiral HPLC: Chiralcel OD column; 99.2:0.8 hexane:isopropanol; $0.6 \mathrm{~mL} / \mathrm{min}$. $[\square]_{\mathrm{D}}{ }^{21}=+3.1^{\circ}(\mathrm{c}=1.00$, $\mathrm{CH}_{2} \mathrm{Cl}_{2}$ ) for an $90 \%$ ee sample. This is a known compound:45 it has previously been established that the $(R)$ enantiomer has a positive sign of rotation. ${ }^{5}$

(4) Takano, D.; Nagamitsu, T.; Ui, H.; Shiomi, K.; Yamaguchi, Y.; Masuma, R.; Kuwajima, I.; Omura, S. Tetrahedron Lett. 1989, 37, 1155.

(5) de Raadt, A.; Fetz, B.; Griengl, H.; Klingler, M. F.; Krenn, B.; Mereiter, K.; Münzer, D. F.; Plachota, P.; Weber, H.; Saf, R. Tetrahedron 2001, 57, 8151. 


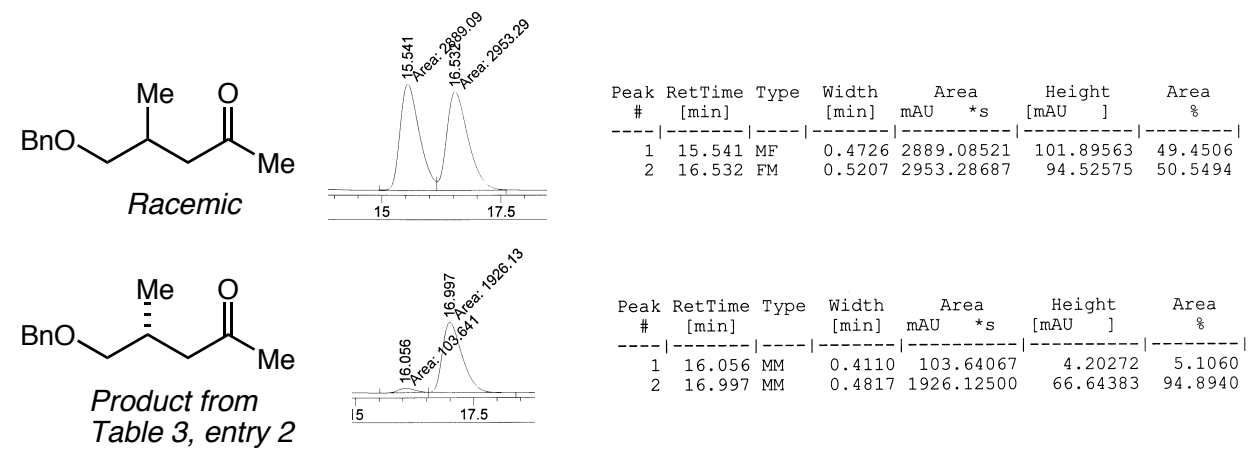

(R)-(+)-4,5-Dimethylhexan-2-one (Scheme 5): Enantiomeric excess was determined by chiral GC: Astec Chiraldex G-TA column; $1.0 \mathrm{~mL} / \mathrm{min}$ He flow, $80{ }^{\circ} \mathrm{C}$. $[\square]_{\mathrm{D}}{ }^{20}=+1.9^{\circ}\left(\mathrm{c}=1.00, \mathrm{CHCl}_{3}\right)$ for a $91 \%$ ee sample. This is a known compound, ${ }^{6}$ the absolute configuration was assigned by analogy.

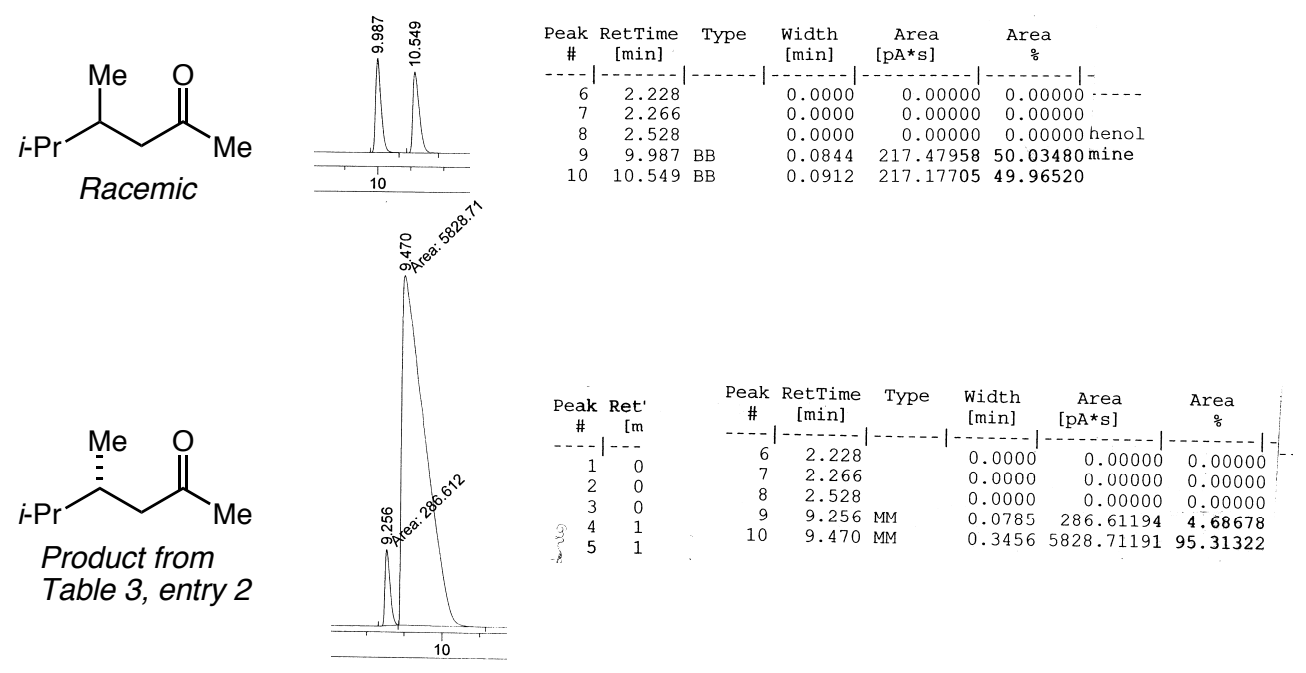

${ }^{1} \mathrm{H}$ NMR spectrum $\left(300 \mathrm{MHz}, \mathrm{C}_{6} \mathrm{D}_{6}\right)$ of ligand 2d:

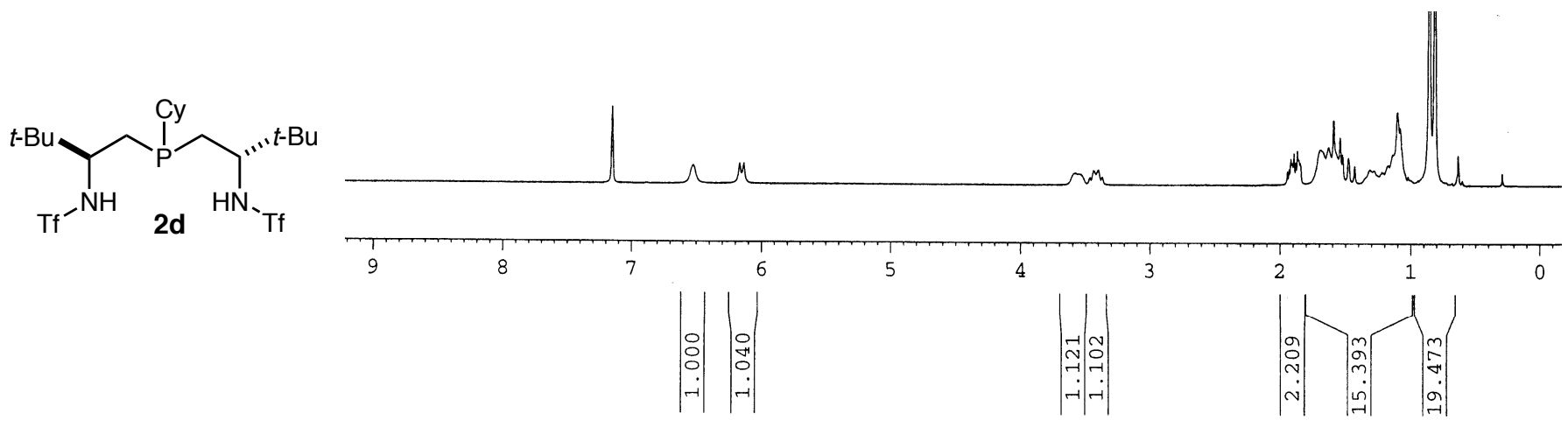

(6) Fraser, P. K.; Woodward, S. Chem. Eur. J. 2003, 9, 779. 
${ }^{1} \mathrm{H}$ NMR spectrum $\left(\mathrm{C}_{6} \mathrm{D}_{6}, 300 \mathrm{MHz}\right)$ of $\mathbf{3}$ :
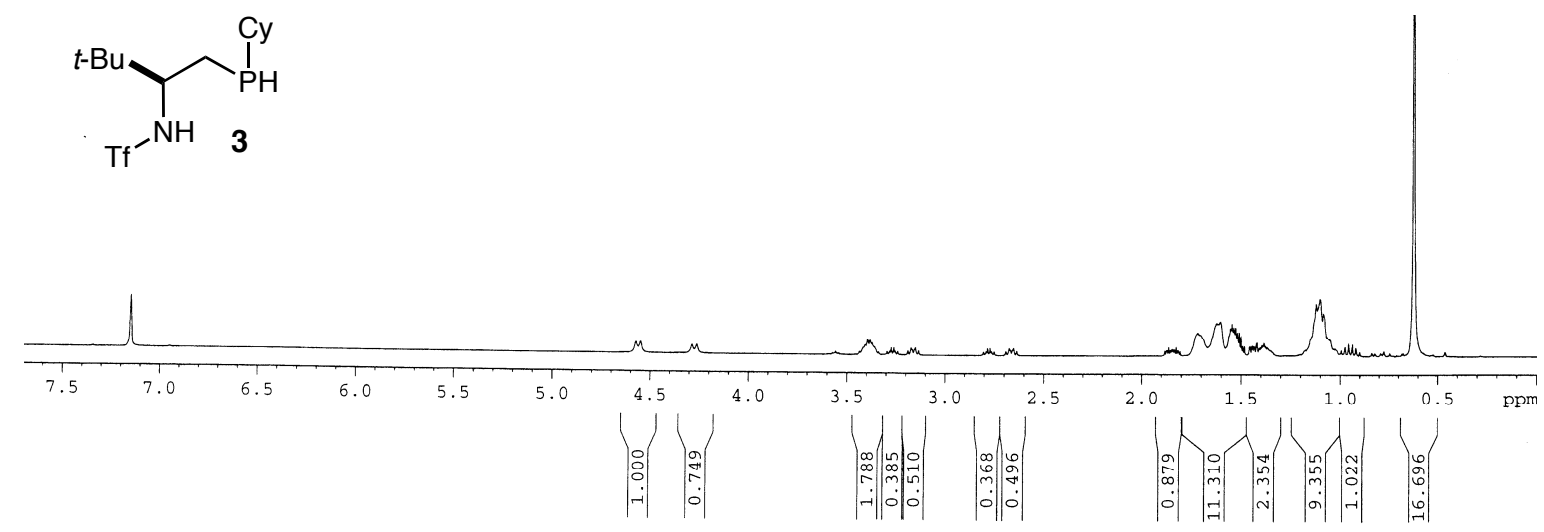

${ }^{1} \mathrm{H}$ NMR spectrum of $(S)-N$-[3,5-bis(trifluoromethyl)benzenesulfonyl]-2-tert-butylaziridine $\left(\mathrm{CDCl}_{3}\right.$, $300 \mathrm{MHz})$ :

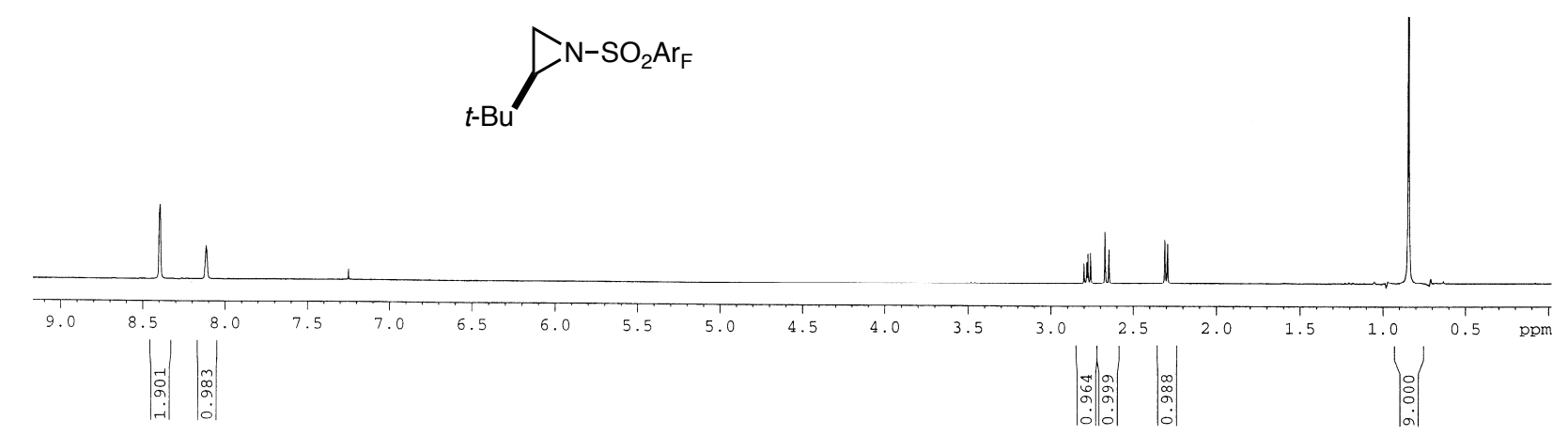

${ }^{1} \mathrm{H}$ NMR spectrum $\left(400 \mathrm{MHz}, \mathrm{CDCl}_{3}\right.$ ) of $\mathbf{4 a}$ :

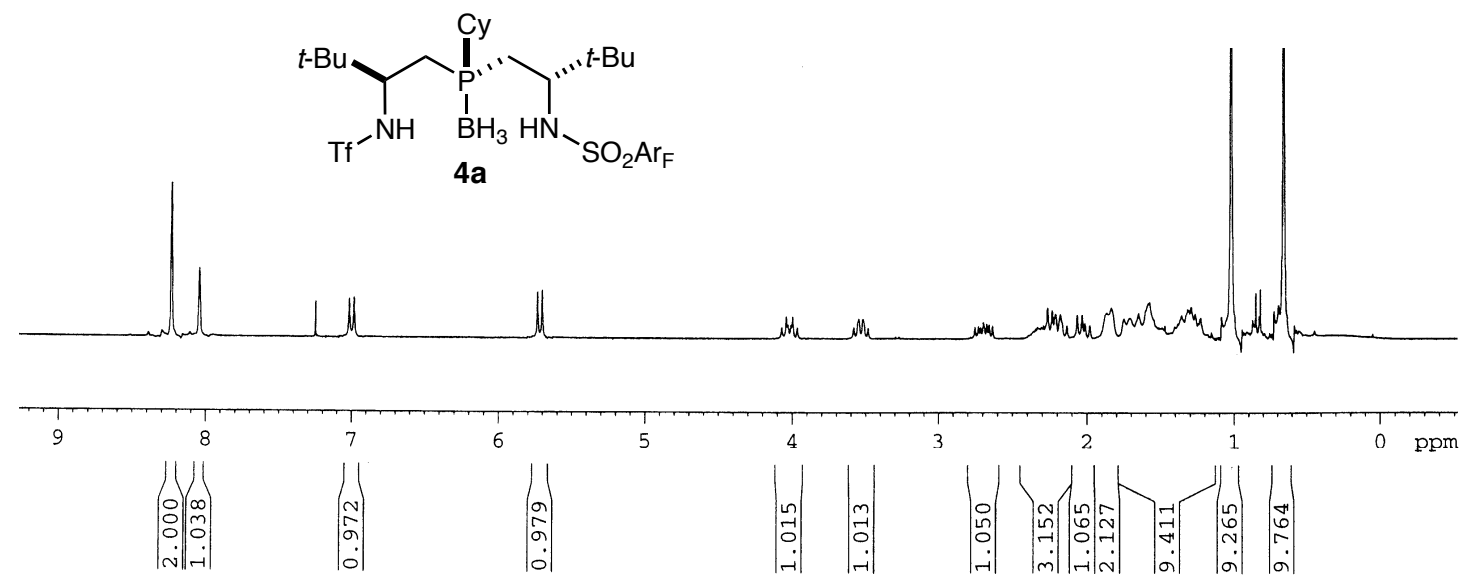


${ }^{1} \mathrm{H}$ NMR $\left(400 \mathrm{MHz}, \mathrm{CDCl}_{3}\right)$ of $\mathbf{4 b}$ :

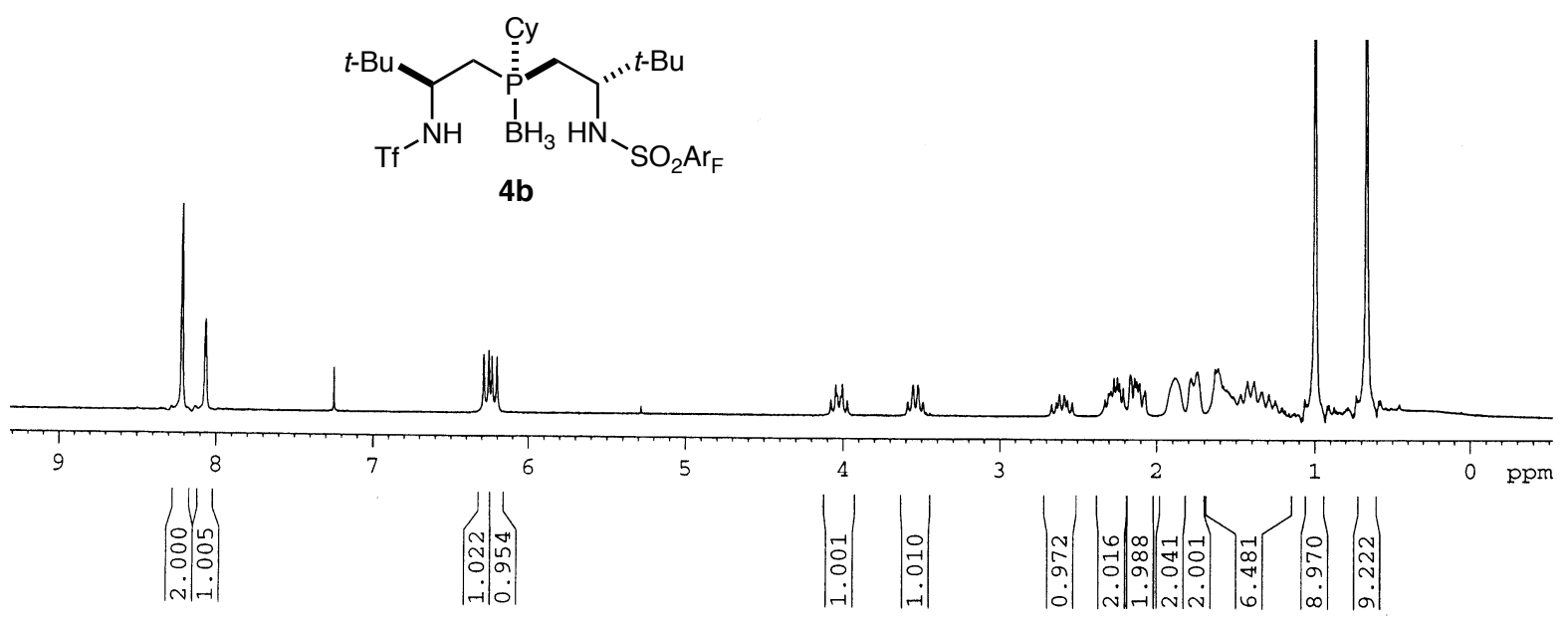

${ }^{1} \mathrm{H}$ NMR (400 MHz, $\mathrm{CDCl}_{3}$ ) of ligand $\mathbf{5 a}$ :

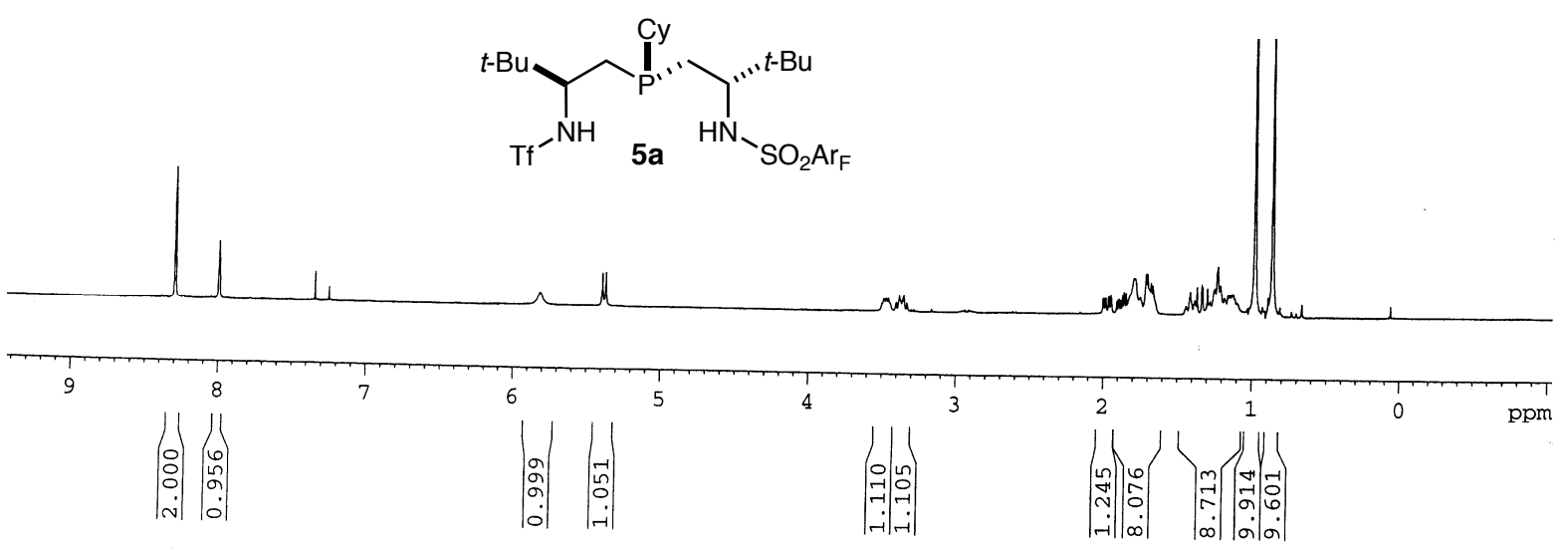

${ }^{1} \mathrm{H}$ NMR (400 MHz, $\mathrm{CDCl}_{3}$ ) of ligand $\mathbf{5 b}$ :
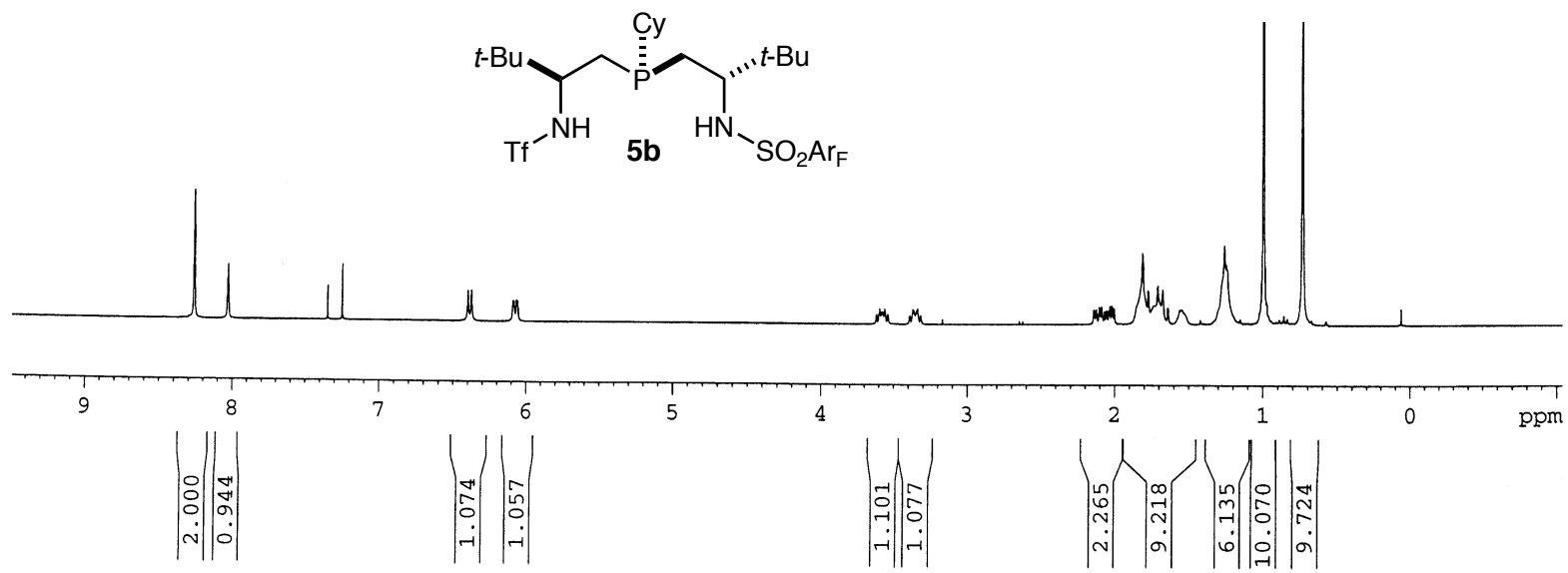
${ }^{1} \mathrm{H}$ NMR $\left(300 \mathrm{MHz}, \mathrm{CDCl}_{3}\right)$ of ligand 6 :

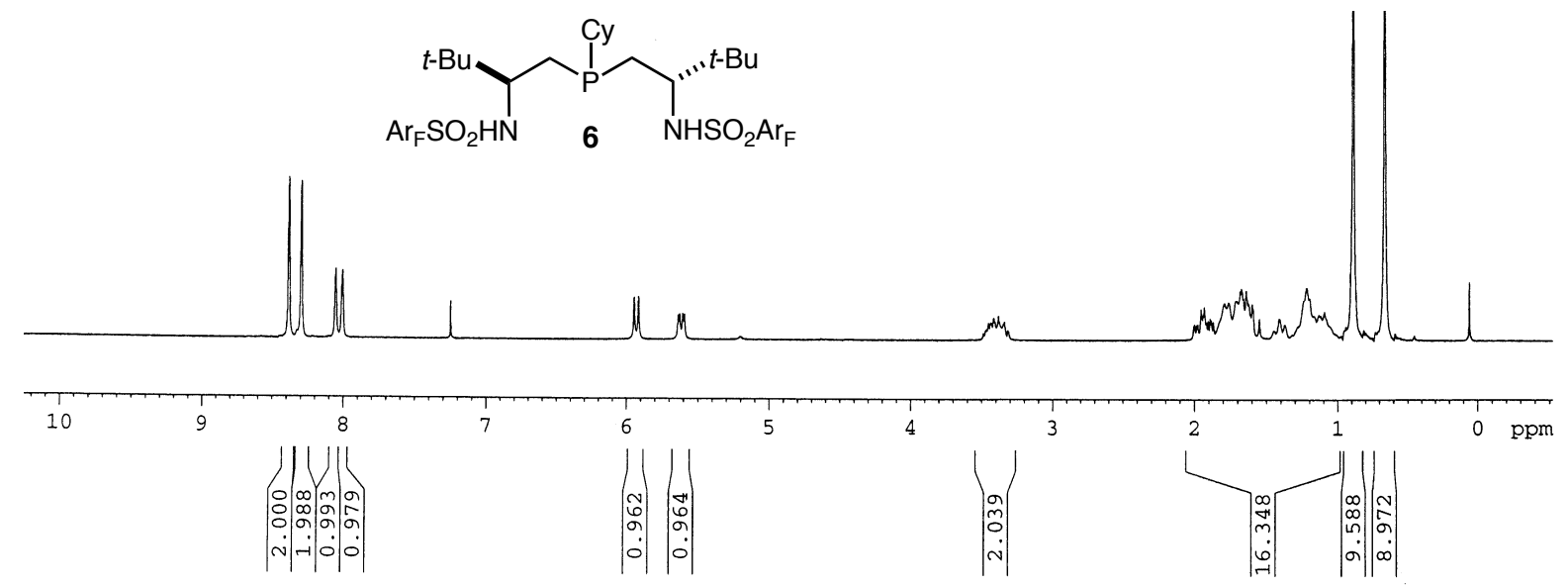

${ }^{1} \mathrm{H}$ NMR spectrum of (+)-4-(Benzyloxymethyl)hexan-2-one (300 $\left.\mathrm{MHz}, \mathrm{CDCl}_{3}\right)$ :<smiles>CC[C@H](COCc1ccccc1)CC(C)=O</smiles>

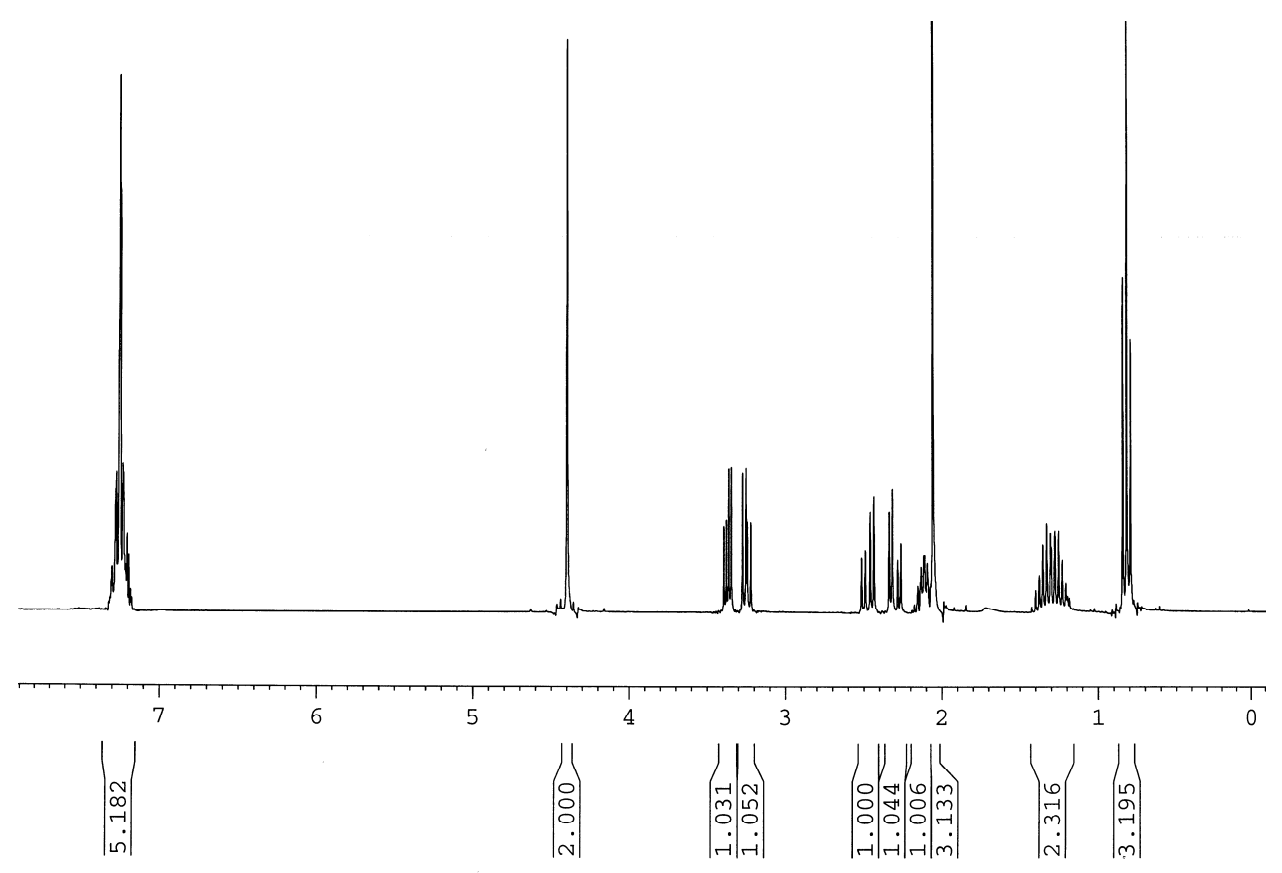


ORTEP diagram illustrating the solid-state structure of $\mathbf{4 b}$ :

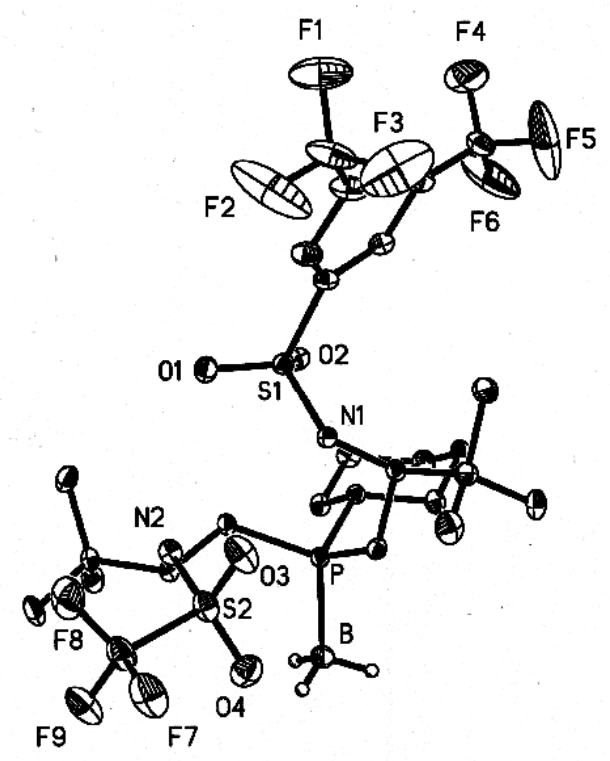

